\section{Desigualdades socioeconômicas em saúde no Brasil: resultados da Pesquisa Mundial de Saúde, 2003}

\author{
Health socioeconomic inequalities in Brazil: \\ results of the World Health Survey, 2003
}

Célia Landmann Szwarcwald 1

Maria do Carmo Leal 2

Giselle Campozana Gouveia 3

Wayner Vieira de Souza 3

\begin{abstract}
Objective: as part of the World Health Organization project of nations' the World Health Survey was performed in Brazil in 2003. This paper has the objective of disclosing social and economic health inequalities through information collected in the Brazilian World Health Survey.

Methods: the population-based survey was conducted in 5000 households selected by probabilistic sampling. To assess social and economic inequalities, three variables were considered: schooling level, number of household belongings and employment status. Logistic regression models were used to establish socioeconomic determinant factors of good self-rated health, as well as periodic gynecologic examination and mammography. As for the health system performance, services were evaluated according to users' expectations.

Results: an outstanding social grading was determined for self-rated health as well as for the coverage of preventive periodic health exams among women. When comparing evaluation of healthcare through payment, "good" rating percentage was always lower for SUS (universal healthcare public system) users for all of the aspects assessed.

Conclusions: healthcare inequalities as demonstrated here should be considered to subsidize public policies and actions of the healthcare system that meet real population needs to overcome social exclusion.
\end{abstract}

Key words Health system, Employee performance appraisal, Socioeconomic factors

\author{
Departamento de Informações em Saúde. Centro de Informação \\ Científica e Tecnológica. Fundação Oswaldo Cruz. Av. Brasil, 4365. \\ Rio de Janeiro, RJ, Brasil. 21.040-360. E-mail: \\ celials@cict.fiocruz.br. \\ 2 Departamento de Epidemiologia. Escola Nacional de Saúde \\ Pública Sérgio Arouca. Fundação Oswaldo Cruz. Rio de Janeiro, RJ, \\ Brasil. \\ ${ }^{3}$ Departamento de Saúde Coletiva. Centro de Pesquisa Aggeu \\ Magalhães (CPqAM). Fundação Oswaldo Cruz Recife, PE, Brasil
}

\section{Resumo}

Objetivo: como parte do projeto da Organização Mundial da Saúde de avaliação de desempenho dos sistemas de saúde das nações, a Pesquisa Mundial de Saúde (PMS) foi realizada no Brasil, em 2003. O presente trabalho tem o objetivo de apresentar algumas evidências de desigualdades socioeconômicas em saúde por meio das informações coletadas na PMS brasileira.

Métodos: o inquérito populacional foi realizado em 5000 domicílios, selecionados por amostragem probabilística. Para examinar as desigualdades socioeconômicas, três variáveis foram consideradas: grau de escolaridade; número de bens do domicílio; e situação de trabalho. Mediante modelos de regressão logística, foram identificados os determinantes da auto-avaliação de saúde boa e das coberturas de exame ginecológico e mamografia. Quanto ao desempenho do sistema de saúde, o atendimento foi avaliado de acordo com as expectativas do usuário.

Resultados: constatou-se acentuado gradiente social no estado de saúde bem como na cobertura dos exames preventivos de saúde entre as mulheres. Comparando-se a avaliação do atendimento por forma de pagamento, $o$ percentual de avaliação "boa" foi sempre menor para os usuários do SUS, para qualquer um dos aspectos avaliados.

Conclusões: as desigualdades em saúde aqui evidenciadas devem ser consideradas para subsidiar as políticas públicas e ações do sistema de saúde que atendam as reais necessidades da população, voltadas à superação da exclusão social.

Palavras-chave Sistema de saúde, Avaliação de desempenho, Fatores socioecômicos 


\section{Introdução}

A avaliação do desempenho dos sistemas de saúde das nações tem obitodo importância crescente entre os gestores do setor saúde, constituindo etapa essencial para o planejamento das ações voltadas para garantir a qualidade da atenção e subsidiar decisões que atendam às reais necessidades da população. Alguns países, como o Canadá, Reino Unido e a Austrália têm desenvolvido, nos últimos anos, instrumentais para a avaliação do desempenho dos sistemas de saúde.

Desde a publicação do "Relatório Black",1 que evidenciou grandes disparidades sociais na situação de saúde na população britânica, e estudos subseqüentes em vários países,2,3 que mostraram uma forte associação entre as condições de saúde e o nível socioeconômico, o monitoramento das desigualdades socioeconômicas em saúde tem sido considerado um componente importante da avaliação do sistema de saúde. As ações e os programas de saúde são avaliados quanto ao seu desempenho em diminuir o gradiente socioeconômico das condições de saúde. 4

No ano 2000, a Organização Mundial da Saúde $(\mathrm{OMS})^{5}$ publicou relatório especificamente dedicado à proposição de uma metodologia para a avaliação de desempenho dos sistemas de saúde dos países membros. Embora com os objetivos louváveis de avaliação desses sistemas de saúde, monitoramento das desigualdades em saúde e da equidade no financiamento da saúde, a metodologia utilizada foi exposta a inúmeras críticas, tanto de cunho metodológico como conceitual, e foi objeto de amplo debate internacional.6-9 As críticas referiram-se não só à falta de utilidade do instrumento para subsidiar a definição das políticas de saúde, mas também à medida de desempenho, combinação matemática de indicadores de distintas dimensões do sistema de saúde para construir um índice composto único, desprovido de qualquer significado. $8,10,11$

Sérios problemas adicionais foram apontados na metodologia da OMS. No que diz respeito ao monitoramento das desigualdades em saúde, o questionamento dirigiu-se, principalmente, à abordagem na construção do indicador utilizado. Uma vez que não foi realizada uma comparação do estado de saúde entre grupos sociais distintos, o índice, baseado em medir variações individuais na probabilidade de morte em crianças até dois anos de idade, era mais influenciado pela desigualdade na distribuição de renda do que pelas ações no âmbito do setor saúde. $12-15$

Um outro grande problema encontrado foi a ausência de dados consistentes, necessários à construção dos indicadores propostos. Em 2001, em fase de reformulação, Organização Mundial da Saúde propôs a aplicação da Pesquisa Mundial de Saúde (PMS), inquérito populacional dirigido à avaliação de desempenho dos sistemas de saúde, nos países membros, adicionando ao instrumento questões relativas ao acesso, à cobertura e à utilização de serviços de saúde. Como parte dessa iniciativa, o governo brasileiro firmou acordo com a OMS para a realização da pesquisa no Brasil. O instrumento originalmente proposto pela OMS foi inteiramente revisto e modificado, desenvolvendo-se a adaptação necessária às particularidades do nosso meio. $\mathrm{O}$ inquérito reformulado foi aplicado em âmbito nacional, no ano de 2003.

O presente trabalho tem o objetivo de avaliar as desigualdades socioeconômicas em saúde, no Brasil, utilizando as informações coletadas na PMS brasileira.

O problema é abordado de forma multidimensional, avaliando-se as desigualdades socioeconômicas do estado de saúde, do acesso e da utilização dos seus serviços, bem como da qualidade da assistência prestada de acordo com as expectativas do usuário.

\section{Métodos}

A Pesquisa Mundial de Saúde foi realizada no Brasil, de janeiro a setembro de 2003, sendo aplicada em 5000 indivíduos com 18 anos e mais de idade, selecionados por amostragem probabilística. O projeto foi aprovado pelo Comitê de Ética em Pesquisa da Fundação Oswaldo Cruz, em dezembro de 2002.

A amostragem foi realizada em três estágios. No primeiro, foram selecionados 250 setores censitários, com probabilidade proporcional ao tamanho, entre todos os setores normais, excluindo-se os setores especiais, tais como as instalações militares, penitenciárias, asilos e reservas indígenas. Situação (urbano ou rural) e porte do município (até 50000; 50000 até $400000 ; 400000$ + habitantes) estratificaram explicitamente as unidades primárias de seleção. A renda média dos chefes dos domicílios do setor foi utilizada para estratificação implícita por nível socioeconômico.

Em cada setor, foram selecionados 20 domicílios, por esquema de amostragem inversa. Em cada domicílio, foi identificado um morador para responder às perguntas relativas às características do domicílio. Apenas um indivíduo do domicílio (selecionado aleatoriamente) respondeu ao questionário 
individual.

Por se tratar de um conjunto de dados obtido por meio de uma amostra complexa, que combina estratificação dos setores censitários, conglomeração e probabilidades de seleção desiguais, as informações sobre o estrato de seleção e o peso amostral foram incorporadas na análise dos dados, que foi realizada com o SUDAAN. 16

A análise estatística foi realizada por faixa etária (18-29; 30-44; 45-59; 60+ anos), sexo, e nível socioeconômico (NSE). Para examinar as desigualdades por NSE, três variáveis foram consideradas: grau de escolaridade (ensino fundamental incompleto; ensino fundamental completo; ensino médio completo ou superior); número de bens do domicílio; e situação de trabalho.

Foi construído um índice, chamado de indicador de bens (IB), calculado por:

$$
I B=\sum_{i}\left(1-f_{i}\right) b_{i}
$$

Onde: i varia de 1 até 10 bens; $b_{i}$ é igual a 1 ou zero respectivamente na presença ou ausência de televisão, geladeira, aparelho de som, telefone fixo, máquina de lavar roupa, telefone celular, automóvel, forno microondas, microcomputador e máquina de lavar louça. O peso atribuído à presença de cada bem do domicílio foi o complementar da freqüência relativa $\left(f_{i}\right)$ de cada bem na amostra total, isto é, tanto mais rara a presença do bem no domicílio, tanto maior o peso atribuído ao bem.

Quanto à situação de trabalho, os indivíduos foram, primeiramente, categorizados entre os que têm ou não trabalho remunerado. No primeiro grupo, os indivíduos foram classificados em trabalhador manual (agricultor ou pescador, trabalhador manual qualificado e não-qualificado) e não-manual (profissional de nível superior, alto funcionário do governo ou empresa, profissional do nível médio, servidor ou funcionário administrativo, comerciário). No segundo grupo, os indivíduos foram classificados em: dona de casa ou do lar; desempregado (procurou trabalho, mas não conseguiu encontrar); aposentado ou incapacitado; outros (estudante, trabalho não remunerado).

A análise do estado geral de saúde foi baseada na seguinte questão: em geral, como o(a) senhor(a) avalia sua saúde atualmente? As respostas variaram em uma escala de 1 a 5 (1=muito ruim; 2=ruim; $3=$ moderada; $4=$ boa; $5=$ muito boa).

Mediante o uso de modelos stepwise de regressão logística multivariada, foram identificados os determinantes socioeconômicos da auto-avaliação de saúde boa (escores $4=$ boa ou 5=muito boa), para cada sexo. Em cada passo, utilizou-se a estatística da razão de verossimilhança para a escolha de variáveis, usando-se como critério de inclusão o nível de significância de 5\% e de exclusão de $10 \%$.

No que se refere ao acesso aos programas de saúde, foram contempladas as coberturas de exame ginecológico entre as mulheres de 18 a 69 anos de idade, e da mamografia, no grupo etário de 40-69 anos. Além das variáveis sócio-demográficas citadas anteriormente, entre as mulheres foram analisadas a situação conjugal (casada ou vive com companheiro; solteira; separada ou divorciada; viúva), tamanho do município de residência (até $50 \mathrm{mil}, 50$ até $400 \mathrm{mil}$, 400 mil e mais habitantes) bem como posse de plano de saúde privado (sim; não).

Objetivando identificar as características das mulheres que realizaram exames periódicos de saúde, adequadamente, para a prevenção do câncer de colo de útero e de mama, foi analisada a associação dos fatores sócio-demográficos com as variáveis realização de exame ginecológico com preventivo há menos de três anos e realização de mamografia há menos de três anos, respectivamente, mediante modelo de regressão logística.

Quanto à avaliação do desempenho do sistema de saúde, foram analisadas a utilização dos serviços de saúde no ano que precedeu à pesquisa e a avaliação do atendimento de acordo com as expectativas do usuário. Para análise dessa última, foram considerados todos os indivíduos que se internaram nos últimos cinco anos (atendimento com internação) ou no caso de ausência de internação nos últimos cinco anos, todos os indivíduos que utilizaram serviços de saúde no último ano (atendimento ambulatorial).

Primeiramente, analisou-se a satisfação do usuário com as habilidades e equipamentos do profissional de saúde bem como com a disponibilidade de medicamentos no atendimento. A seguir, utilizando-se uma escala de um a cinco, avaliou-se o grau de satisfação com os seguintes aspectos: tempo de deslocamento; tempo de espera; tratamento respeitoso; intimidade respeitada; clareza nas explicações dadas pelos profissionais de saúde; tempo para fazer perguntas; possibilidade de obter informações; participação na tomada de decisões; falar em privacidade com profissionais de saúde; sigilo das informações pessoais; liberdade de escolha do profissional de saúde; limpeza das instalações; espaço disponível das salas de espera e de exames. No caso de assistência com internação, consideraram-se, adicionalmente, "facilidade em ter visitas de familiares e amigos" e "contato com mundo exterior". A avaliação do atendimento foi realizada de acordo com a forma de pagamento: não pagou 
(Sistema Único de Saúde, SUS); através do plano de saúde ou direto, sem reembolso (não SUS).

Para efeitos da análise estatística, calculou-se um índice de avaliação do atendimento, atribuindo-se um ponto para cada resposta "muito boa" ou "boa" relativa à avaliação de cada um dos aspectos do atendimento, e utilizou-se escala variando de 1 a 100. A avaliação do atendimento foi realizada de acordo com a forma de pagamento: não pagou (SUS); através do plano de saúde; direto, sem reembolso.

Na avaliação da assistência por parte do usuário, considerou-se ainda se ele se sentiu alguma vez discriminado (foi tratado pior) por algum dos seguintes motivos: falta de dinheiro, classe social, sexo, idade, raça /cor, nacionalidade ou tipo de doença.

\section{Resultados}

Dentre os 4997 entrevistados que auto-avaliaram a saúde, $9 \%$ avaliaram como "ruim" ou "muito ruim",
$53 \%$ como "boa" ou muito "boa", e $38 \%$ como "moderada". A percepção de saúde foi pior entre as mulheres quando comparadas aos homens: o percentual de auto-avaliação "boa" ou muito "boa" foi de $47 \%$, para o sexo feminino, e de $60 \%$, para o sexo masculino. Uma grande amplitude de variação do percentual de auto-avaliação "boa" ou muito "boa" foi encontrada por faixa etária, variando de $28 \%$, entre os indivíduos com 60 anos e mais de idade, a 70\%, entre os jovens de 18 a 29 anos (Tabela 1).

Ainda pelos dados dispostos na Tabela 1, evidencia-se a variação da percepção de saúde "boa" ou "muito boa" por grau de escolaridade. O percentual mostrou-se tanto menor quanto pior o nível de instrução, variando de $41 \%$, entre os entrevistados com ensino fundamental incompleto, a $72 \%$, entre aqueles com ensino médio completo ou superior, incluindo-se nesta categoria os indivíduos com formação universitária, correspondendo a $6,2 \%$ do total da amostra.

De forma geral, o padrão encontrado é o de percepção pior da saúde entre os mais idosos, entre

\section{Tabela 1}

Percentual de indivíduos com auto-avaliação da saúde "boa" ou "muito boa" segundo faixa etária, sexo e grau de escolaridade. Brasil, 2003.

\begin{tabular}{|c|c|c|c|c|c|}
\hline \multirow[t]{2}{*}{ Sexo } & \multirow[t]{2}{*}{ Faixa etária } & \multicolumn{3}{|c|}{ Grau de escolaridade } & \multirow[t]{2}{*}{ Total } \\
\hline & & $\begin{array}{l}\text { Fundamental } \\
\text { incompleto }\end{array}$ & $\begin{array}{l}\text { Fundamental completo e } \\
\text { ensino médio incompleto }\end{array}$ & $\begin{array}{l}\text { Ensino médio } \\
\text { completo }\end{array}$ & \\
\hline \multirow[b]{3}{*}{ Feminino } & $18-29$ & 51,5 & 64,0 & 75,9 & 64,7 \\
\hline & $30-44$ & 39,6 & 54,4 & 73,2 & 52,5 \\
\hline & $45-59$ & 25,2 & 39,7 & 51,8 & 32,2 \\
\hline \multirow{5}{*}{ Masculino } & $18-29$ & 65,8 & 78,4 & 83,0 & 75,2 \\
\hline & $30-44$ & 57,8 & 65,3 & 76,8 & 64,9 \\
\hline & $45-59$ & 45,3 & 61,5 & 68,5 & 53,1 \\
\hline & $60+$ & 27,9 & 45,8 & 45,1 & 31,4 \\
\hline & Total & 49,4 & 69,8 & 75,3 & 60,2 \\
\hline \multirow{2}{*}{ Total } & $18-29$ & 58,5 & 71,1 & 78,9 & 69,6 \\
\hline & $30-44$ & 47,8 & 59,4 & 74,8 & 58,1 \\
\hline
\end{tabular}


Determinantes sócio-demográficos da auto-avaliação de saúde" boa" ou "muito boa". Brasil, 2003.

Modelo I

\begin{tabular}{|c|c|c|c|c|c|}
\hline \multirow[t]{2}{*}{ Variável } & & \multicolumn{2}{|c|}{ Sexo feminino } & \multicolumn{2}{|c|}{ Sexo masculino } \\
\hline & & $\operatorname{Exp}(b)$ & IC95\% & $\operatorname{Exp}(b)$ & IC95\% \\
\hline \multirow[t]{2}{*}{ Idade } & & $0,969 * *$ & $0,96-0,98$ & 0,968 ** & $0,96-0,97$ \\
\hline & Fundamental incompleto & $0,304 * *$ & $0,24-0,38$ & 0,410 ** & $0,31-0,54$ \\
\hline \multirow[t]{2}{*}{ Grau de escolaridade } & Ensino médio incompleto & $0,532 * *$ & $0,40-0,71$ & 0,691 ** & $0,49-0,97$ \\
\hline & Ensino médio completo & 1,000 & - & 1,000 & - \\
\hline
\end{tabular}

Modelo II

\begin{tabular}{|c|c|c|c|c|c|}
\hline \multirow[t]{2}{*}{ Variável } & & \multicolumn{2}{|c|}{ Sexo feminino } & \multicolumn{2}{|c|}{ Sexo masculino } \\
\hline & & $\operatorname{Exp}(b)$ & IC95\% & $\operatorname{Exp}(b)$ & IC95\% \\
\hline \multirow[t]{2}{*}{ Idade } & & $0,964 * *$ & $0,96-0,97$ & $0,965 * *$ & $0,96-0,97$ \\
\hline & Fundamental incompleto & $0,458 * *$ & $0,37-0,57$ & $0,584 * *$ & $0,42-0,81$ \\
\hline \multirow[t]{2}{*}{ Grau de escolaridade } & Ensino médio incompleto & $0,655 * *$ & $0,49-0,87$ & $0,830 * *$ & $0,58-1,18$ \\
\hline & Ensino médio completo & 1,000 & - & 1,000 & - \\
\hline \multicolumn{2}{|l|}{ Indicador de bens } & $1,372 * *$ & $1,26-1,49$ & $1,248 * *$ & $1,11-1,40$ \\
\hline \multicolumn{6}{|c|}{ Modelo III } \\
\hline \multirow[t]{2}{*}{ Variável } & & \multicolumn{2}{|c|}{ Sexo feminino } & \multicolumn{2}{|c|}{ Sexo masculino } \\
\hline & & $\operatorname{Exp}(b)$ & IC95\% & $\operatorname{Exp}(b)$ & IC95\% \\
\hline \multirow[t]{2}{*}{ Idade } & & $0,968 * *$ & $0,96-0,98$ & $0,968 * *$ & $0,96-0,98$ \\
\hline & Fundamental incompleto & $0,476 * *$ & $0,37-0,61$ & 0,710 & $0,50-1,01$ \\
\hline \multirow[t]{2}{*}{ Grau de escolaridade } & Ensino médio incompleto & $0,657 * *$ & $0,49-0,88$ & 0,985 & $0,68-1,44$ \\
\hline & Ensino médio completo & 1,000 & - & 1,000 & - \\
\hline \multirow[t]{3}{*}{ Indicador de bens } & & $1,346 * *$ & $1,23-1,47$ & $1,177 * *$ & $1,04-1,33$ \\
\hline & Trabalhador não manual & 1,000 & - & 1,000 & - \\
\hline & Trabalhador manual & 0,884 & $0,63-1,24$ & $0,547 * *$ & $0,39-0,76$ \\
\hline \multirow[t]{3}{*}{ Trabalho } & Dona de casa & 0,862 & $0,66-1,13$ & - & - \\
\hline & Desempregado & 0,946 & $0,66-1,36$ & $0,586 *$ & $0,39-0,89$ \\
\hline & Aposentado / Incapacitado & 0,701 & $0,46-1,07$ & $0,4524 * *$ & $0,30-0,68$ \\
\hline
\end{tabular}

$\star(p<0,05), * *(p<0,01)$

as mulheres quando são comparadas com os homens em qualquer faixa etária, e entre os de pior nível de instrução relativamente aos de grau de escolaridade mais elevado. Além disso, constata-se que o gradiente social na auto-avaliação de saúde "boa" ou "muito boa" se repete para qualquer faixa de idade ou sexo (Tabela 1).

A análise dos dados por situação de trabalho indica que quase metade (49\%) dos indivíduos de 18 anos e mais de idade não têm trabalho remunerado. Para o sexo feminino, esse percentual é de $63 \%$, e para o masculino, de $33 \%$, sendo essa diferença 
Proporção (\%) de mulheres que fizeram exame preventivo para câncer de colo de útero e mamografia há menos de três anos segundo as características sócio-demográficas. Brasil, 2003.

\begin{tabular}{|c|c|c|c|c|c|}
\hline \multirow[t]{2}{*}{ Variáveis } & Categorias & \multicolumn{2}{|c|}{ Preventivo para câncer de colo de útero } & \multicolumn{2}{|c|}{ Mamografia* } \\
\hline & & $\%$ & $p$ & $\%$ & $p$ \\
\hline & $<50 \mathrm{mil}$ & 56,8 & \multirow{3}{*}{$<0,001$} & 34,2 & \multirow{3}{*}{$<0,001$} \\
\hline \multirow[t]{2}{*}{ Tamanho do município } & 50 até $400 \mathrm{mil}$ & 67,5 & & 44,1 & \\
\hline & $400 \mathrm{mil}+$ & 72,3 & & 62,3 & \\
\hline \multirow{4}{*}{$\begin{array}{l}\text { Faixa etária } \\
\text { (anos) }\end{array}$} & $18-29$ & 53,1 & \multirow{4}{*}{$<0,001$} & - & - \\
\hline & $30-39$ & 77,1 & & - & - \\
\hline & $40-49$ & 75,6 & & 45,5 & \multirow{2}{*}{ NS } \\
\hline & $50-69$ & 62,2 & & 48,6 & \\
\hline \multirow{2}{*}{$\begin{array}{l}\text { Teve ou não filhos (entre } \\
\text { mulheres de } 18-29 \text { anos) }\end{array}$} & Já teve filhos & 64,5 & \multirow{2}{*}{$<0,001$} & - & - \\
\hline & Não teve filhos & 40,8 & & - & - \\
\hline \multirow{2}{*}{$\begin{array}{l}\text { Grau de } \\
\text { escolaridade }\end{array}$} & Fundamental incompleto & 60,7 & \multirow{2}{*}{$<0,001$} & 36,6 & \multirow{2}{*}{$<0,001$} \\
\hline & Fundamental completo+ & 70,9 & & 70,3 & \\
\hline \multirow{2}{*}{ Cor da pele } & Branca & 67,3 & \multirow{2}{*}{0,026} & 54,3 & \multirow{2}{*}{$<0,001$} \\
\hline & Outra & 63,5 & & 39,5 & \\
\hline \multirow{2}{*}{ Situação conjugal } & Casada / Companheiro & 72,4 & \multirow{2}{*}{$<0,001$} & 49,2 & \multirow{2}{*}{0,080} \\
\hline & Não vive com companheiro & 54,9 & & 43,9 & \\
\hline \multirow{2}{*}{$\begin{array}{l}\text { Tem trabalho } \\
\text { remunerado }\end{array}$} & Sim & 70,7 & \multirow{2}{*}{$<0,001$} & 53,2 & \multirow{2}{*}{0,002} \\
\hline & Não & 62,0 & & 43,6 & \\
\hline \multirow{3}{*}{$\begin{array}{l}\text { Número de bens } \\
\text { domicílio }\end{array}$} & $0-3$ & 53,4 & \multirow{3}{*}{$<0,001$} & 21,6 & \multirow{3}{*}{$<0,001$} \\
\hline & $4-7$ & 67,1 & & 49,2 & \\
\hline & $8+$ & 81,1 & & 75,2 & \\
\hline \multirow{2}{*}{ Plano de saúde } & Sim & 82,1 & \multirow{2}{*}{$<0,001$} & 72,3 & \multirow{2}{*}{$<0,001$} \\
\hline & Não & 59,6 & & 36,7 & \\
\hline
\end{tabular}

* Somente para mulheres entre 40 e 69 anos de idade.

explicada pela grande proporção de mulheres donas de casa. Em geral, $11 \%$ declararam estar procurando emprego sem conseguir (desempregados), com pequena diferença entre os sexos. Entre as pessoas com trabalho remunerado, $57 \%$ são trabalhadores manuais, $35 \%$ são profissionais do nível médio, e $8 \%$ do nível superior.

Em relação à distribuição dos indivíduos por presença de bens no domicílio, observou-se que o bem mais freqüentemente encontrado é a televisão $(91,8 \%)$, seguida da geladeira $(89,9 \%)$ e do aparelho de som $(78,2 \%)$. Com proporções variando de 30 a $60 \%$, foram encontrados o telefone fixo $(57,4 \%)$, a máquina de lavar roupa $(56,4 \%)$, o telefone celular $(37,9 \%)$ e o automóvel $(35,1 \%)$. Os bens mais raramente encontrados foram o forno de microondas $(21,3 \%)$, o computador $(15,6 \%)$ e a máquina de lavar-louça $(4,8 \%)$. As freqüências relativas aqui descritas serviram como base de cálculo do indicador de bens (IB), conforme explicitado na seção de metodologia.

Os resultados dos modelos de regressão logística 
tendo a auto-avaliação de saúde boa ou muito boa como variável resposta, aplicados separadamente para cada sexo, podem ser analisados na Tabela 2. O modelo I considera as desigualdades por grau de escolaridade, controlando-se o efeito da idade. Verifica-se que as diferenças no nível de instrução, quando comparadas ao grupo com ensino médio completo são significativas para ambos os sexos, com gradiente mais pronunciado entre as mulheres. Tomando-se como categoria-controle o ensino médio completo, o odds-ratio (OR) entre as mulheres com ensino fundamental incompleto é de 0,30 e, entre os homens, de 0,41, mesmo após o ajuste pela idade.

Os resultados do modelo II mostram efeito positivo do indicador de bens do domicílio (construído tal como explicitado na seção de metodologia), sugerindo uma melhor avaliação da saúde entre as pessoas com maior riqueza, ainda que se controlando os efeitos do grau de escolaridade e da idade (Tabela 2). Nota-se que para o sexo masculino, o indicador de bens foi mais importante que o nível de instrução para explicar o gradiente socioeconômico da autoavaliação de saúde boa.

O modelo III considera adicionalmente os efeitos da situação de trabalho. Resultados bem diferentes são encontrados por sexo (Tabela 2). Entre as mulheres, a questão do trabalho não se mostrou relevante, para quaisquer dos indicadores incluídos no modelo. Já entre os homens, a situação de trabalho foi fundamental. Juntamente com o indicador de bens do domicílio, as variáveis relativas à situação de trabalho explicaram o gradiente socioeconômico da resposta, tornando os efeitos do grau de escolaridade não significativos. Os resultados do modelo III indicam efeitos negativos e significativos para as categorias compostas por homens trabalhadores manuais, desempregados, e aposentados ou incapacitados, quando comparadas à categoria de trabalhadores não manuais.

$\mathrm{Na}$ Tabela 3, apresentam-se as diferenças sóciodemográficas na proporção de mulheres que realizam, dentro de um período de três anos, exame preventivo para câncer de colo de útero e de mama. No caso de exame ginecológico adequado, os dados mostram que os grupos formados por mulheres de 30 a 49 anos, que têm trabalho remunerado, vivem com companheiro, e residem em cidades grandes, são os que têm as maiores coberturas. Além disso, os resultados evidenciam acentuadas desigualdades por nível socioeconômico, seja esse mensurado por grau de instrução, ou número de bens do domicílio. Igualmente, a cobertura é significativamente maior entre as mulheres que possuem um plano de saúde privado. Quanto ao exame de mamografia, as carac- terísticas sócio-demográficas são semelhantes, mas o gradiente social é ainda mais acentuado: a cobertura varia de $22 \%$, entre as mulheres com menos de quatro bens no domicílio, a $75 \%$, entre aquelas que possuem oito ou mais bens.

Os resultados da pesquisa indicaram que, aproximadamente, $24 \%$ dos brasileiros têm plano de saúde privado. Analisando-se por categoria de número de bens, o percentual de pessoas com plano de saúde varia de $4 \%$, entre os de pior NSE, a $64 \%$, entre os de melhor condição socioeconômica.

Entre os 5000 indivíduos que participaram da pesquisa, $70 \%$ precisaram de assistência de saúde no último ano, sendo a utilização mais freqüente entre os que possuem planos de saúde $(81 \%)$ do que entre os que não possuem (67\%).

Dos 5000 entrevistados, 1544 (31\%) relataram assistência de saúde com internação nos últimos cinco anos antes da pesquisa. Desses, $71,0 \%$ foram atendidos pelo SUS, 22,5\% pagaram através do plano de saúde e $6,5 \%$ pagaram direto, sem reembolso. Entre os demais participantes, 2388 (69\%) tiveram pelo menos um atendimento ambulatorial no ano que antecedeu à entrevista. Desses, 19\% pagou diretamente o atendimento (sem reembolso), $21 \%$ pagou através do plano e $60 \%$ utilizou o SUS.

Os aspectos relacionados à avaliação do atendimento ambulatorial (Tabela 4), revelaram que o "tempo de espera até ser atendido" teve o menor grau de satisfação por parte dos usuários, dentre todos os aspectos analisados. Já os aspectos que dizem respeito à intimidade, ao sigilo das informações pessoais e as habilidades dos profissionais de saúde, obtiveram percentuais de satisfação elevados.

Comparando-se o percentual de usuários que fizeram uma avaliação "boa" ou "muito boa" por forma de pagamento do atendimento, invariavelmente para todos os aspectos estudados, o percentual é sempre menor para os usuários do SUS (Tabela 4). Entre esses, menos da metade fez uma avaliação positiva do "tempo de espera" e da "liberdade de escolha do profissional de saúde" (49\%). O índice médio de avaliação do atendimento ambulatorial, em uma escala de 0 a 100, foi de 77, variando de 70 , entre os usuários do SUS, a 89, entre os que pagaram o atendimento através do plano de saúde.

Dentre todos os aspectos da internação hospitalar (Tabela 4), o menor percentual de satisfação correspondeu à "liberdade de escolha do profissional de saúde", enquanto que os aspectos relacionados à disponibilidade de medicamentos, equipamentos adequados e respeito à intimidade foram apontados como os de maior satisfação.

A análise comparativa das avaliações dos 
Percentual de indivíduos com auto-avaliação da saúde "boa" ou "muito boa" segundo faixa etária, sexo e grau de escolaridade. Brasil, 2003.

\begin{tabular}{|c|c|c|c|}
\hline \multicolumn{4}{|c|}{ Atendimento Ambulatorial } \\
\hline \multirow[t]{2}{*}{ Aspectos avaliados } & \multicolumn{2}{|c|}{ Forma de pagamento } & \multirow{2}{*}{$\begin{array}{l}\text { Total } \\
\mathrm{n}=961\end{array}$} \\
\hline & $\begin{array}{c}\text { Não SUS } \\
(n=961)\end{array}$ & $\begin{array}{c}\text { SUS } \\
(n=1427)\end{array}$ & \\
\hline & $\%$ & $\%$ & $\%$ \\
\hline Tempo de deslocamento & 78,2 & 68,8 & 72,6 \\
\hline Tempo de espera até ser atendido & 73,4 & 45,4 & 56,7 \\
\hline Respeito no tratamento & 96,6 & 86,0 & 90,2 \\
\hline Respeito à intimidade & 97,1 & 93,9 & 95,2 \\
\hline Clareza das explicações & 93,5 & 80,1 & 85,5 \\
\hline Tempo para fazer perguntas & 90,2 & 65,9 & 75,7 \\
\hline Possibilidade de obter informações sobre tratamentos alternativos & 85,8 & 60,1 & 70,4 \\
\hline Participação nas decisões sobre a conduta & 85,2 & 59,8 & 70,0 \\
\hline Privacidade com os profissionais de saúde & 94,3 & 83,3 & 87,7 \\
\hline Sigilo das informações pessoais & 96,7 & 89,1 & 92,2 \\
\hline Liberdade de escolha do profissional de saúde & 87,7 & 49,0 & 64,6 \\
\hline Limpeza das instalações & 97,6 & 79,2 & 86,6 \\
\hline Espaço das salas de espera e de exames & 88,0 & 63,9 & 73,6 \\
\hline Habilidades do profissional de saúde satisfatórias & 96,7 & 90,3 & 92,9 \\
\hline Equipamentos adequados & 96,6 & 87,7 & 91,3 \\
\hline Disponibilidade de medicamentos adequada & 88,2 & 75,9 & 80,8 \\
\hline \multicolumn{4}{|c|}{ Internação hospitalar } \\
\hline \multirow[t]{3}{*}{ Aspectos avaliados } & \multicolumn{2}{|c|}{ Forma de pagamento } & Total \\
\hline & $\begin{array}{l}\text { Não SUS } \\
(\mathrm{n}=447)\end{array}$ & $\begin{array}{c}\text { SUS } \\
(n=1096)\end{array}$ & $\mathrm{n}=1544$ \\
\hline & $\%$ & $\%$ & $\%$ \\
\hline Tempo de deslocamento & 79,3 & 60,7 & 66,1 \\
\hline Tempo de espera até ser atendido & 83,3 & 67,9 & 72,4 \\
\hline Respeito no tratamento & 93,1 & 85,8 & 87,9 \\
\hline Respeito à intimidade & 96,5 & 90,3 & 92,1 \\
\hline Clareza das explicações & 89,6 & 78,3 & 81,6 \\
\hline Tempo para fazer perguntas & 86,5 & 63,0 & 69,8 \\
\hline Possibilidade de obter informações sobre tratamentos alternativos & 83,9 & 58,3 & 65,7 \\
\hline Participação nas decisões sobre a conduta & 82,8 & 59,1 & 65,9 \\
\hline Privacidade com os profissionais de saúde & 89,8 & 68,0 & 74,3 \\
\hline Sigilo das informações pessoais & 94,9 & 81,5 & 85,5 \\
\hline Liberdade de escolha do profissional de saúde & 82,9 & 47,3 & 57,5 \\
\hline Limpeza das instalações & 91,2 & 78,1 & 81,9 \\
\hline Espaço das salas de espera e de exames & 90,7 & 71,6 & 77,1 \\
\hline Possibilidade de receber visitas & 87,1 & 70,0 & 75,0 \\
\hline Contato com o mundo exterior & 81,5 & 59,2 & 65,7 \\
\hline Habilidades do profissional de saúde satisfatórias & 94,5 & 90,0 & 91,3 \\
\hline Equipamentos adequados & 97,0 & 90,4 & 92,3 \\
\hline Disponibilidade de medicamentos adequada & 97,8 & 90,8 & 92,9 \\
\hline
\end{tabular}

$\mathrm{n}$ = número de indivíduos pesquisados 
Percentual (\%) de indivíduos que sofreram algum tipo de discriminação segundo o tipo de assistência e forma de pagamento. Brasil, 2003.

\begin{tabular}{|c|c|c|c|}
\hline \multicolumn{4}{|c|}{ Atendimento Ambulatorial } \\
\hline \multirow[t]{3}{*}{ Aspectos avaliados } & \multicolumn{2}{|c|}{ Forma de pagamento } & \multirow[t]{2}{*}{ Total } \\
\hline & Não SUS & SUS & \\
\hline & $\%$ & $\%$ & $\%$ \\
\hline Discriminação por sexo & 1,4 & 1,4 & 1,4 \\
\hline Discriminação por idade & 1,5 & 2,0 & 1,8 \\
\hline Discriminação por falta de dinheiro & 5,2 & 11,1 & 8,7 \\
\hline Discriminação por classe social & 5,2 & 9,5 & 7,8 \\
\hline Discriminação por cor & 1,0 & 1,4 & 1,2 \\
\hline Discriminação por tipo de doença & 1,3 & 2,0 & 1,7 \\
\hline Discriminação por nacionalidade & 0,0 & 0,3 & 0,2 \\
\hline \multicolumn{4}{|c|}{ Internação hospitalar } \\
\hline \multirow[t]{3}{*}{ Aspectos avaliados } & \multicolumn{2}{|c|}{ Forma de pagamento } & Total \\
\hline & Não SUS & sus & \\
\hline & $\%$ & $\%$ & $\%$ \\
\hline Discriminação por sexo & 3,1 & 2,3 & 2,5 \\
\hline Discriminação por idade & 2,1 & 3,7 & 3,2 \\
\hline Discriminação por falta de dinheiro & 8,0 & 14,9 & 12,9 \\
\hline Discriminação por classe social & 7,2 & 13,1 & 11,4 \\
\hline Discriminação por cor & 1,7 & 1,4 & 1,5 \\
\hline Discriminação por tipo de doença & 2,4 & 2,7 & 2,6 \\
\hline Discriminação por nacionalidade & 0,0 & 0,4 & 0,3 \\
\hline
\end{tabular}

Os percentuais foram calculados para os grupos Sistema único de Saúde (SUS) e não SUS sobre o total de indivíduos que receberam assistência ambulatorial ou tiveram internação hospitalar.

usuários por forma de pagamento da internação indica que os percentuais de satisfação entre os usuários do SUS são sempre inferiores aos obtidos entre os usuários que pagaram o atendimento através do plano de saúde, para qualquer dos aspectos contemplados na pesquisa. O índice médio de avaliação da internação, em uma escala de 0 a 100, foi de 73 , para o total de usuários; de 88 , para aqueles que pagaram a internação através do plano de saúde; e de 68, para os usuários do SUS.

Uma proporção significativa de usuários se sentiu discriminada no atendimento ambulatorial: $9 \%$ sentiram que lhe trataram pior do que às outras pessoas por falta de dinheiro e $8 \%$ devido à classe social (Tabela 5). Entre os usuários do SUS, esses percentuais foram ainda maiores, de $11 \%$ e $10 \%$, respectivamente. Do total de usuários, $1,2 \%$ se sentiu discriminado pela cor.

Igualmente entre os entrevistados com internação nos últimos cinco anos, uma fração considerável se sentiu discriminada, em níveis mais elevados ainda do que os usuários do atendimento ambulatorial: $13 \%$ por falta de dinheiro e $11 \%$ pela classe social. Entre àqueles não pagaram pela internação, $15 \%$ e $13 \%$ sentiram que foram tratados pior por estes dois motivos, respectivamente (Tabela 5).

\section{Discussão}

A utilidade da auto-avaliação de saúde deriva da sua validade, refletida, em parte, pela relação com as condições clínicas, bem como com indicadores de morbidade e mortalidade. 17 Existem também evidên- 
cias da sua habilidade de predizer respostas clínicas importantes, que vão além do escopo de indicadores objetivos. 18,19

Várias teorias têm sido sugeridas para explicar o poder de predição da auto-avaliação de saúde em relação à morbidade e à mortalidade futura. Primeiramente, a percepção ruim da saúde pode ser uma indicação do processo saúde-doença, ainda não detectável biológica ou medicamente. ${ }^{17}$ Em outra vertente explicativa, a auto-avaliação da saúde envolve o conhecimento do próprio indivíduo sobre uma ampla variedade de fatores influentes era sua vida, tais como: comportamentos de risco, história familiar de doenças, longevidade familiar, entre outros, ${ }^{20}$ de tal forma que a percepção da própria saúde é considerada como um bom marcador do estado de saúde da população.

Os resultados deste trabalho mostram claramente a existência de associação entre fatores socioeconômicos e a auto-avaliação de saúde na população brasileira. Os achados são consistentes aos encontrados nos países desenvolvidos.21,22 Um estudo recente mostrou gradientes sociais pronunciados em onze países europeus (Bélgica, Dinamarca, França, Alemanha, Grã-Bretanha, Grécia, Irlanda, Itália, Holanda, Portugal e Espanha), baseando-se em três indicadores de saúde: auto-avaliação do estado de saúde; limitação das atividades devido a um problema de saúde; e doença de longa duração ou incapacidade. Os resultados foram, invariavelmente, mais desfavoráveis quanto pior a condição social dos indivíduos. 2

Na presente análise, marcadamente, a posse de bens foi um fator de grande contribuição à percepção satisfatória da saúde, tanto entre as mulheres como entre os homens. Conforme o discutido por Martikainenet et al., ${ }^{23} \mathrm{o}$ indicador de riqueza não só reflete as necessidades materiais da vida, tais como a possibilidade de adquirir boa nutrição e habitação adequada, mas é também um marcador de bem-estar social.

Em relação aos outros determinantes socioeconômicos da auto-avaliação de saúde boa, diferenciais importantes foram encontrados por sexo. Para os homens, a situação de trabalho, incluindo o tipo de ocupação e o desemprego, bem como a posse de bens materiais foram fatores essenciais para explicar as diferenças da percepção da saúde por NSE. Para as mulheres, a instrução incompleta e a privação material foram as variáveis mais associadas.

No que se refere à análise das desigualdades socioeconômicas da cobertura do exame para prevenção do câncer de colo de útero, foram encontradas diferenças importantes por grau de escolari- dade e número de bens do domicílio, além das desigualdades de acesso por tamanho de município. Entretanto, não se pode deixar de registrar que uma parcela significativa das mulheres brasileiras está se beneficiando desse serviço, com intervalo de tempo adequado entre os exames. Sabendo-se que o câncer de colo de útero apresenta um grande potencial de cura, sobretudo quando diagnosticado e tratado precocemente, ${ }^{24}$ esse resultado é bastante positivo.

Inversamente, os resultados da PMS brasileira revelaram que a cobertura do exame de mamografia é baixa, abrangendo pouco menos da metade das mulheres brasileiras entre 40 e 69 anos de idade. Ainda que, no Brasil, a faixa etária recomendada para fazer o exame de rotina é de 50 a 69 anos, mais de $40 \%$ das mulheres desse grupo etário revelaram que nunca tinham feito esse exame. Foram encontrados gradientes importantes por tamanho de município, possivelmente em decorrência da grande concentração de serviços de diagnósticos médicos nas capitais e municípios de grande porte. Segundo informações da Pesquisa Assistência MédicoSanitária, de 2002, as capitais detêm $43 \%$ dos mamógrafos, mas somente $26 \%$ da população feminina de 40 a 69 anos de idade. 25

Ainda em relação à mamografia, observou-se também um pronunciado diferencial por forma de financiamento desse tipo de serviço. A cobertura alcança $72 \%$ no grupo de mulheres que tem plano de saúde, no mesmo nível de utilização da população feminina norte-americana, 26 mas o dobro da encontrada no grupo de mulheres que utilizam a assistência pública. Apenas recentemente o Ministério da Saúde passou a recomendar a realização da mamografia como exame de escolha para o diagnóstico do câncer de mama, o que pode ser um dos fatores explicativos para as baixas coberturas no sistema público de saúde.

Desigualdades de acesso ao diagnóstico de câncer de colo de útero e de mama são bem documentadas na literatura internacional. De modo geral, são associadas ao baixo nível de escolaridade, ${ }^{27}$ ao desequilíbrio geográfico na distribuição dos serviços, 28 e se acentuam em função do poder de compra do usuário, seja por meio do plano privado ou desembolso direto. 29 Apesar do seu caráter universal, o SUS carece, ainda hoje, de mecanismos, de fato eficientes, de modo a fazer com que determinados serviços, disponíveis em municípios de maior porte, possam se tornar também acessíveis à população residente em municípios de menor porte e de pior nível socioeconômico.

Em relação à avaliação da assistência de saúde, do ponto de vista do usuário do serviço, a análise dos 
dados da PMS brasileira apontou que a satisfação com o atendimento é relacionada à qualidade do tratamento por parte dos profissionais de saúde, ao cuidado recebido e à solução do problema de saúde. Os aspectos que apresentaram menor grau de satisfação foram relativos aos serviços de saúde, seja no que diz respeito ao acesso, seja com as instalações do estabelecimento de saúde em que a assistência foi prestada, sobretudo entre os indivíduos que utilizaram o SUS.

Os resultados da presente análise corroboram os achados de outros estudos no Brasil, que evidenciaram a deterioração da qualidade dos serviços de saúde públicos, como, por exemplo, o trabalho de Assis et al. 30 que revelou que as principais queixas relacionadas ao atendimento médico foram: demora no atendimento; várias idas ao local de atendimento para conseguir o serviço; e a falta de garantia de conseguir os exames ou mesmo de continuidade de tratamento.

A PMS brasileira revelou, ainda, que os principais problemas identificados pelos usuários foram os mesmos nos dois grupos, os que pagaram e os que não pagaram pelo serviço prestado. Porém, o grau de satisfação com a assistência pública foi menor, invariavelmente. Segundo Assis et al.,30 o Brasil convive com acessos seletivos, excludentes e focalizados, fato que se relaciona às desigualdades sociais na distribuição de benefícios, tendo como conseqüência a deterioração da qualidade da atenção prestada nos serviços públicos.

\section{Referências}

1. Townsend P, Davidson N. Inequalities in health: the black teport and the health divide. Harmondworth: Penguin; 1982.

2. Mackenbach JP, Kunst AE, Cavelaars AEJM, Gronhof F, Geurts JJM. Socioeconomic inequalities in morbidity and mortality in Western Europe. The EU Working Group on Socioeconomic Inequalities in Health. Lancet 1997; 349: 1655-9.

3. Macintyre S. The Black Report and beyond: what are the issues? [review]. Soc Sci Med 1997; 44: 723-45.

4. Braveman P, Tarimo E. Social inequalities in health within countries: not only an issue for affluent nations. Soc Sci Med 2002; 54: 1621-35.

5. WHO (World Health Organization). The World Health report 2000.Geneva; 2000.

6. Häkkinen U, Ollila E, editors. The World Health Report 2000: What does it tell us about health systems? Analyses by Finnish experts. Helsinki: National Research and Development Center for Welfare and Health (STAKES). [online]. [http://www.stakes.fi/english/publicati/ Publications.htm], 2000. [2005 Jan 27].
Um outro grave problema identificado na análise dos dados da PMS foi o alto percentual de pessoas que se sentiram discriminadas, independentemente da forma de pagamento do atendimento. Observouse, ainda, que o sentimento de discriminação foi um dos principais motivos de insatisfação, em qualquer uma das dimensões da assistência prestada. As principais formas de discriminação apontadas pelos respondentes foram: a falta de dinheiro e a classe social, sendo importante notar que os percentuais de indivíduos que se sentiram tratados pior por motivo relacionados à exclusão social foram sempre maiores para os usuários do SUS, em comportamento oposto aos princípios que regem o sistema de saúde brasileiro.

Em suma, os dados da PMS trouxeram para o plano nacional algumas questões concernentes às desigualdades em saúde, que podem subsidiar a reorientação da organização dos serviços no Brasil. $\mathrm{O}$ inquérito proporcionou informações relevantes para o aprimoramento da avaliação do desempenho do sistema nacional de saúde e o monitoramento das desigualdades socioeconômicas na situação de saúde, bem como no acesso e utilização dos serviços. As investigações de cada aspecto falam, claramente, a favor da manutenção da abordagem multidimensional, que permite delinear um panorama mais próximo da complexidade do objeto e subsidiar decisões que atendam as reais necessidades da população, voltadas à superação da exclusão social.

7. Navarro V. Assessment of the World Health Report 2000. Lancet 2000; 36: 1598-601.

8. Rosén M. Can the WHO Health Report improve the performance of health systems? Scand J Public Health 2001; 29: 76-80.

9. Ugá A, Almeida C, Szwarcwald CL, Travassos C, Viacava F, Ribeiro JM, Costa NR, Buss PM, Porto S. Considerations on methodology used in the World Health Organization 2000: Report. Cad Saúde Pública 2001; 17: 705-12.

10. Navarro V. The World Health Report 2000: can health care systems be compared using a single measure of performance? Am J Public Health 2002; 92: 31-4.

11. Almeida C, Braveman P, Gold MR, Szwarcwald CL, Ribeiro JM, Miglionico A, Millar JS, Porto S, Costa NR, Rubio VO. Methodological concerns and recommendations on policy consequences of the World Health Report 2000. Lancet 2001; 357: 1692-7.

12. Szwarcwald CL. On the World Health Organisation's measurement of health inequalities. J Epidemiol Community Health 2002; 56: 177-82. 
13. Braveman P, Starfield B, Geiger HJ. World Health Report 2000: how it removes equity from the agenda for public health monitoring and policy. Br Med J 2001; 323: 678-81.

14. Howelling TAJ, Kunst AE, Mackenbach JP. World Health Report 2000: inequality index and socioeconomic inequalities in mortality. Lancet 2001; 357: 1671-72.

15. Wolfson M, Rowe G. On measuring inequalities in health. Bull World Health Organ 2001;79: 553-60.

16. Shah BV, Barnwell BG, Bieler GS. SUDAAN user's manual. Release 7.5. Research Triangle Park, NC: Research Triangle Institute; 1997.

17. Eriksson I, Unden AL, Elofson S. Self-rated health. Comparisons between three different measures. Results from a population study. Int J Epidemiol 2001; 30: 326-33.

18. Bergner M, Rohman ML. Health status measures: an overview and guide for selection. Ann Rev Public Health 1987; 8: 191-210.

19. Idler EL, Benyamini Y. Self-rated health and mortality: a review of twenty-seven community studies. J Health Soc Behav 1997; 38: 21-37.

20. Manor O, Matthews S, Power C. Dichotomous or categorical response? Analysing self-rated health and lifetime social class. Int J Epidemiol 2000; 29: 149-57.

21. Franks P, Gold MR, Fiscella K. Sociodemographics, selfrated health, and mortality in the US. Soc Sci Med 2003; 56: $2505-14$

22. Heistaro S, Vartiainen E, Puska P. Trends in self-rated health in Finland 1972-1992. Prev Med 1996; 25: 625-32.

Recebido em 11 de julho de 2005

Versão final apresentada em 18 de novembro de 2005

Aprovado em 21 de novembro de 2005
23. Martikainen P, Adda J, Ferrie JE, Smith GD, Marmot M. Effects of income and health on GHQ depression and poor self rated health in white collar women and men in the Whitehall II study. J Epidemiol Community Health 2003; 57: 718-23.

24. Sankaranarayanan R, Budukh AM, Rajkumar R. Effective screening programmes for cervical cancer in low-and middle-income developing countries [review]. Bull World Health Organ 2001; 79: 954-62.

25. DATASUS. Pesquisa Assistência Médico-Sanitária, 2002. [online].http://tabnet.datasus.gov.br/cgi/ams/msopcao.htm [2005 jan 27].

26. Coughlin SS, Uhler RJ, Bobo JK, Caplan L. Breast cancer screening practices among women in the United States, 2000. Cancer Causes Control 2004; 15: 159-70.

27. Borras JM, Guillen M, Sanchez V, Junca S, Vicente R. Educational level, voluntary private health insurance and opportunistic cancer screening among women in Catalonia (Spain). Eur J Cancer Prev 1999; 8: 427-34.

28. Agurto I, Bishop A, Sanchez G, Betancourt Z, Robles S. Perceived barriers and benefits to cervical cancer screening in Latin America. Prev Med 2004; 39: 91-8.

29. Hayward RA, Shapiro MF, Freeman HE, Corey CR. Who gets screened for cervical and breast cancer? Results from a new national survey. Arch Intern Med 1988; 148: 1177-81.

30. Assis MMA, Villa TCS, Nascimento, MAA. Acesso aos serviços de saúde: uma possibilidade a ser construída na prática. Cienc Saúde Coletiva 2003; 8: 815-23. 